\title{
INFLUENCIA DEL COLOR EN EL COMPORTAMIENTO ALIMENTARIO DE Geochelone (Chelonoidis) carbonaria (Spix 1824) EN CAUTIVERIO
}

\author{
COLOR INFLUENCE ON THE FEEDING BEHAVIOR OF Geochelone (Chelonoidis) \\ carbonaria (SPIX 1824) IN CAPTIVITY
}

\author{
DE LA OSSA, V. JAIME ${ }^{1 *}$ Dr., GUEVARA-RIAÑO LISSETTE² Zootec., HORTA- \\ OROZCO LUISA D ${ }^{2}$ Zootec., MARTINEZ-MENDOZA DAISSY ${ }^{2} Z_{\text {Zootec. }}$ \\ ${ }^{1 *}$ Profesor Universidad de Sucre, Facultad de Ciencias Agropecuarias, Colombia. \\ Grupo de Investigación en Biodiversidad Tropical. \\ ${ }^{2}$ Zootecnistas Universidad de Sucre, Colombia. \\ ${ }^{*}$ Correspondencia: jaimedelaossa@yahoo.com
}

\section{Resumen}

Se evaluó la influencia del color de diez especies de flores en el comportamiento alimentario de Geochelone (Chelonoidis) carbonaria bajo condiciones de manejo ex situ, utilizándose un grupo de 20 ejemplares adultos constituido por 11 hembras y 9 machos, divididos en dos lotes. Se registraron manifestaciones de comportamiento enfatizando en el tiempo de acceso al alimento y tiempo de consumo. Se analizan aspectos alimentarios mediante el grado de preferencia respecto a la coloración de seis especies de flores de color rojo, amarillo y blanco y cuatro especies de color rojo y amarillo, en dos tratamientos, fresco y seco. Para el tiempo de acceso al alimento no se encontró diferencia significativa en cuanto al color y la especie de flor, indicando así que los animales accedían al alimento con la misma rapidez, independientemente del color. Para el parámetro tiempo de consumo se halló diferencia altamente significativa para el efecto color y especie en presentación fresca.

Palabras clave: alimentación, coloración, flores, Geochelone (Chelonoidis) carbonaria

\section{Abstract}

The present study evaluated the influence of color in ten flower species in the nourishing behavior of Geochelone (Chelonoidis) carbonaria under handling conditions "ex situ", with a group of 20 adult individuals comprised by 11 females and 9 males divided in two lots. Behavior manifestations were recorded emphasizing the time of access to the food and time of consumption. Nourishing aspects were analyzed through the preference grade regarding the coloration of six flower species 
of red, yellow and white color and four species of red and yellow color, in two treatments, fresh and dry. For the parameter time of access to the food, no significant difference was found regarding flower color and species, thus indicating that animals acceded to the food with the same speed, regardless the color. For the parameter time of consumption, a highly significant difference was found for the effect color and species in the fresh appearance.

Key words: food "nourishing", colorations, flowers, Geochelone (Chelonoidis) carbonaria.

\section{Introducción}

Señala LEGLER (1963) que al Oeste de Colombia y al Este de Panamá G. carbonaria ocupa hábitat forestales; CASTAÑO-MORA y LUGO (1979) indican que es una especie terrestre que prefiere las sabanas, montes de galería, pequeños bosques y moriches (agrupaciones de Mauritia sp.) y se tiene como una especie más adaptada a regiones en donde hay una prolongada estación seca.

Algunos autores como CASTAÑO y LUGO (1979), MERCHAN et al, (1998), COLVÉE (2002) condujeron estudios de biología en G. carbonaria que tratan sobre los aspectos morfológicos, reproductivos, de organización social y alimentación; sobre este último aspecto las investigaciones registran que los morrocoyes tienen una preferencia alimenticia por los frutos maduros, flores, hojas verdes y carroña.

En general para G. carbonaria, Según diversos autores los frutos silvestres forman parte de la dieta; específicamente, MEDEM (1962) registra el consumo de material vegetal principalmente, pero que los adultos son también parcialmente carroñeros; por su parte FREIBERG (1971) anota que esta especie puede pacientemente esperar bajo un árbol los frutos maduros, y podría consumir los frutos caídos; FRETEY (1977) complementa que la dieta está compuesta principalmente de vegetación y quizá invertebrados; MOSKOVITS (1981) identificó varias especies de plantas preferidas por $G$. carbonaria en un área de bosque húmedo natural en el Estado de Roraima, Brasil. Estas incluyeron Duquetia surinamensis (Annonaceae), Annona sp. (Annonaceae), Spondia lutea (Anacardiaceae), Genipa americana (Rubiaceae) y Bagassa guianenesis (Moraceae).

CASTAÑO y LUGO (1979) observaron que en cautiverio los alimentos preferidos son las plantas pequeñas, especialmente los retoños, frutas maduras, flores de colores vistosos (rojas, amarillas, etc.), hortalizas, hojas verdes, carne y pescado frescos o descompuestos, sobras de comida, excrementos propios o de otros animales; apetecen menos las frutas verdes, los cítricos, tubérculos y plantas acuáticas; G. carbonaria come alimentos concentrados para aves o ratas; igualmente, PRITCHARD y TREBBAU (1984) anotan que, según propias 
observaciones, los morrocoyes tiene una predilección por las flores de color rojo y amarillo.

Adicionalmente STRONG y FRAGOSO (2006) de manera complementaria indican que el rol de G. carbonaria como dispersor de semillas en el noroeste de Brasil, mediante análisis de muestras fecales, es amplio, llegando a detectar 11 especies de plantas en este proceso, lo cual demuestra su importante contribución en el mantenimiento de la diversidad y estructura forestal, así como la conformación y predilección de dieta.

Para el género puede anotarse que poseen un comportamiento alimentar típicamente herbívoro, con ingestión de material vegetal abundante y diverso, además de ser considerados fundamentales dispersores de semillas. Para Geochelone pardalis se registra el consumo de por lo menos 75 especies de herbáceas, suculentas y leñosas, de este porcentaje las flores, frutos y semillas llegan al 67\% siendo a la vez un eficiente dispersor de semillas de Aizoaceae, Chenopodiaceae, Crassulaceae, Cyperaceae, Fabaceae, Poaceae y Scrophulariaceae (MILTON, 1992). Geochelone gigantea, por su parte, se conoce como consumidor de herbáceas y arbóreas, siendo también un importante dispersor de semillas, que puede ingerir casi la totalidad de la flora terrestre de su hábitat (HNATIUK, 1978); VARELA y BUCHER (2002) indican que Geochelone chilensis es un amplio consumidor de frutas y también un importante dispersor de semillas.

En este trabajo se demuestra la importancia que tiene el color del alimento en la selección que de él hace $G$. carbonaria, y se reconoce que existe diferenciación del color para esta especie y se discute la posibilidad diferencial entre visón cromática y acromática desde un análisis del comportamiento de selección del alimento.

\section{Materiales y Métodos}

El estudio se realizó en la granja El Perico, Universidad de Sucre, municipio de Sampués a $9^{\circ} 12^{\prime} \mathrm{N}$ y $75^{\circ} 26^{\prime} \mathrm{O}$. Se utilizó un grupo de 20 ejemplares de $G$. carbonaria (morrocoy), constituido por 11 hembras y 9 machos, divididos en dos lotes, fueron mantenido en cautividad en dos encierros de $75 \mathrm{~m}^{2}(10 \mathrm{~m} \times 7,5 \mathrm{~m})$ cada uno. En el lote número uno había cinco machos y cinco hembras, y en el lote número dos había cuatro machos y seis hembras, con densidad media de 0,13 ind $/ \mathrm{m}^{2}$. Cada encierro estuvo equipado con dos estanques de $1 \mathrm{~m}^{2}$, para proporcionar agua que les permitiera beber y bañarse a voluntad (Fig. 1). 


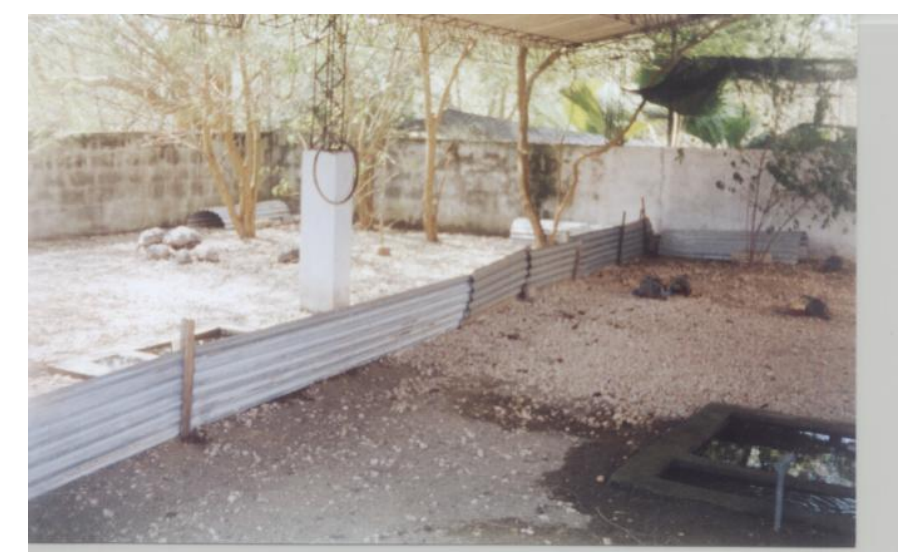

Figura 1. Vista general de los encierros experimentales para G. carbonaria.

La recolección de información se llevó a cabo mediante la aplicación de tablas para registros, en donde se consignaba tiempo de acceso al alimento y tiempo de consumo, una vez que este era suministrado, El tiempo fue tomado mediante cronómetro (Seiko $\mathrm{S} 143 \AA$ ). La identificación de los animales fue dada por marcación con tinta indeleble no tóxica en la parte dorsal y costal del carapax (DE LA OSSA, 1999) con números del 1 al 20, asignándoles de 1 al 11 a las hembras y del 12 al 20 a los machos.

Para evaluar los aspectos etológicos se midió el grado de atracción respecto a la coloración de las distintas especies de flores ofrecidas como alimento, como el tiempo que se demoraban en consumirlo. Se utilizaron diez especies, así: Hibiscus rosa-sinensis (bonche), Bougainvillea glabra (trinitaria), loxa cocinea (coral), Rosa $s p$ (rosa), Dianthus sp. (clavel), Aster spp. (margarita) con los colores rojo, amarillo y blanco, y Lantana spp. (lantana), Tithonia diversifolia (margarita criolla), Acacia farnesiana (acacia) y Chrysanthemum spp. (fuggi), con las variedades rojo y amarillo (Fig.2). Todas las flores fueron suministradas en dos tratamientos: en el lote número uno flores frescas y en el lote número dos flores secas, al $2 \%$ del peso vivo de los animales cada tres días (Tabla 1); el alimento fue ofrecido siempre entre las 9:00 y las 10:00, y colocado en dos puntos equidistantes del encierro a una distancia media de $5 \mathrm{~m}$ respecto de las paredes.

Después de cada tratamiento por especie de flor se les daba a los animales un alimento normal al $4 \%$ del peso vivo compuesto por frutas maduras: Musa $s p$. (banano), Mangifera indica (mango), Citrus sinensis (naranja), Crescentia cujete (totumo); vegetales frescos como Guazuma ulmifolia (guácimo), Periskia bleo (bleo de puerco), Portulaca sp. (verdolaga), Lactuca sativa (lechuga), Brassica oleracea (repollo); carne de pescado y de res, siendo el componente vegetal el $80 \%$ y el componente animal el $20 \%$. 


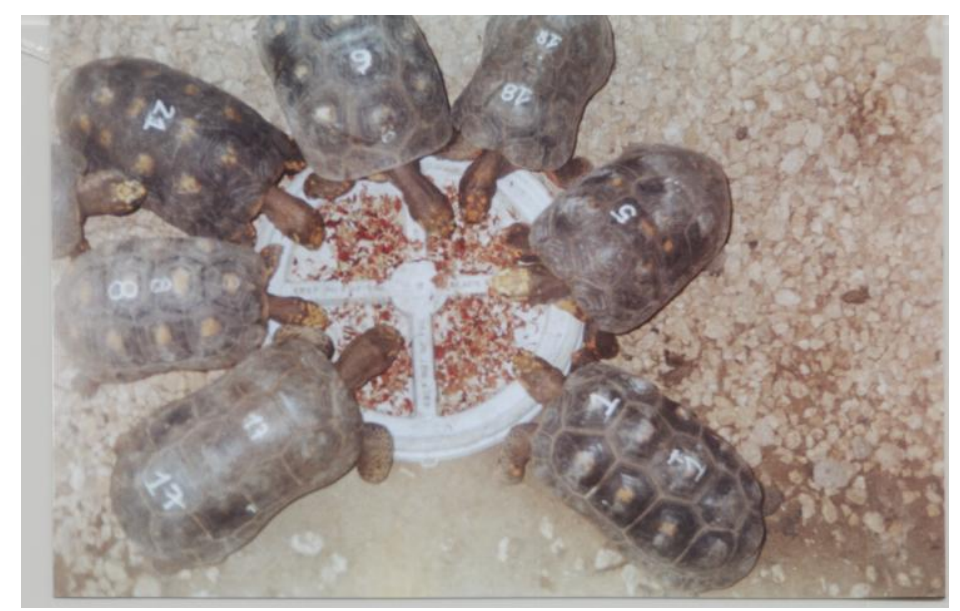

Figura 2. Ingesta de G. carbonaria en los ensayos de aceptación de color.

Se utilizó un diseño completamente al azar con arreglo factorial en cada uno de los ensayos; en el primer ensayo de seis por tres y en el segundo de cuatro por dos; se aplicó ANOVA para comparar medias en color y tiempo, y una prueba Tukey para comparaciones múltiples de medias de aquellas variables que fueron significativas (ZAR, 1996).

Tabla 1. Esquema de la oferta del alimento

\begin{tabular}{|l|l|l|l|l|l|}
\hline Nombre Científico & Nombre Común & Día & Lote 1 & Lote 2 & $\%$ \\
\hline Hibiscus rosa-sinensis & bonche rojo & 1 & Fresco & Seco & 2 \\
\hline Hibiscus rosa-sinensis & bonche blanco & 3 & Fresco & Seco & 2 \\
\hline Hibiscus rosa-sinensis & bonche amarillo & 6 & Fresco & Seco & 2 \\
\hline Mezcla de Hibiscus rosa-sinensis & Mezcla de bonche & 9 & Fresco & Seco & 2 \\
\hline & Alimento normal & 12 & -- & -- & 4 \\
\hline Bougainvillea glabra & trinitaria roja & 15 & Fresco & Seco & 2 \\
\hline Bougainvillea glabra & trinitaria amarilla & 18 & Fresco & Seco & 2 \\
\hline Bougainvillea glabra & trinitaria blanca & 21 & Fresco & Seco & 2 \\
\hline Mezcla de Bougainvillea glabra & Mezcla de trinitarias & 24 & Fresco & Seco & 2 \\
\hline & Alimento normal & 27 & -- & -- & 4 \\
\hline loxa cocinea & coral roja & 30 & Fresco & Seco & 2 \\
\hline loxa cocinea & coral amarillo & 33 & Fresco & Seco & 2 \\
\hline loxa cocinea & coral blanco & 36 & Fresco & Seco & 2 \\
\hline Mezcla de loxa cocinea & Mezcla de corales & 39 & Fresco & Seco & 2 \\
\hline & Alimento normal & 42 & -- & -- & 4 \\
\hline Rosa sp. & rosa roja & 45 & Fresco & Seco & 2 \\
\hline Rosa sp. & rosa amarilla & 48 & Fresco & Seco & 2 \\
\hline Rosa sp. & rosa blanca & 51 & Fresco & Seco & 2 \\
\hline Mezcla de Rosa sp. & Mezcla de rosas & 54 & Fresco & Seco & 2 \\
\hline & Alimento normal & 57 & -- & -- & 4 \\
\hline Aster spp. & margarita roja & 60 & Fresco & Seco & 2 \\
\hline Aster spp. & margarita amarilla & 63 & Fresco & Seco & 2 \\
\hline Aster spp. & margarita blanca & 66 & Fresco & Seco & 2 \\
\hline Mezcla de Aster spp. & Mezcla de margaritas & 69 & Fresco & Seco & 2 \\
\hline & Alimento normal & 72 & -- & -- & 4 \\
\hline Dianthus sp. & clavel roja & 75 & Fresco & Seco & 2 \\
\hline Dianthus sp. & clavel amarilla & 78 & Fresco & Seco & 2 \\
\hline
\end{tabular}




\begin{tabular}{|l|l|l|l|l|l|}
\hline Dianthus sp. & clavel blanco & 81 & Fresco & Seco & 2 \\
\hline Mezcla de Dianthus sp. & Mezcla de clavel & 84 & Fresco & Seco & 2 \\
\hline & Alimento normal & 87 & -- & -- & 4 \\
\hline Lantana spp. & lantana amarilla & 90 & Fresco & Seco & 2 \\
\hline Lantana spp. & lantana roja & 93 & Fresco & Seco & 2 \\
\hline Mezcla de Lantana spp. & Mezcla de lantana & 96 & Fresco & Seco & 2 \\
\hline & Alimento Normal & 99 & -- & -- & 4 \\
\hline Tithonia diversifolia & margarita criolla amarilla & 102 & Fresco & Seco & 2 \\
\hline Tithonia diversifolia & margarita criolla roja & 105 & Fresco & Seco & 2 \\
\hline Mezcla de Tithonia diversifolia & Mezcla de margarita criolla & 108 & Fresco & Seco & 2 \\
\hline & Alimento normal & 111 & -- & -- & 4 \\
\hline Chrysanthemum spp. & fuggi rojo & 114 & Fresco & Seco & 2 \\
\hline Chrysanthemum spp. & fuggi amarillo & 117 & Fresco & Seco & 2 \\
\hline Mezcla de Chrysanthemum spp. & Mezcla de crisantemo & 120 & Fresco & Seco & 2 \\
\hline & Alimento normal & 123 & -- & -- & 4 \\
\hline Acacia farnesiana & acacia roja & 126 & Fresco & Seco & 2 \\
\hline Acacia farnesiana & acacia amarilla & 129 & Fresco & Seco & 2 \\
\hline Mezcla de Acacia farnesiana & Mezcla de acacia & 132 & Fresco & Seco & 2 \\
\hline & Alimento normal & 135 & -- & -- & 4 \\
\hline
\end{tabular}

\section{Resultados}

El tiempo de acceso al alimento en presentación fresco y seco no tuvo diferencias significativas, las medias son estadísticamente iguales; indicando así, que los animales accedían al alimento con la misma rapidez, independientemente del color (Tabla 2).

Tabla 2. Medias y desviaciones estándar en minutos por colores para tiempo de acceso al alimento.

\begin{tabular}{|c|c|c|c|c|c|c|c|}
\hline \multicolumn{4}{|c|}{ Fresco } & \multicolumn{4}{|c|}{ Seco } \\
\hline Color & $\mathbf{n}$ & $\mathbf{X}$ & $\sigma$ & Color & $\mathbf{n}$ & $\bar{X}$ & $\sigma$ \\
\hline Rojo & 52 & $2,914 \mathrm{a}$ & 14,048 & Rojo & 53 & $7,646 \mathrm{a}$ & 142,396 \\
\hline Amarillo & 50 & $2,176 \mathrm{a}$ & 4,946 & Amarillo & 47 & $3,873 \mathrm{ab}$ & 51,291 \\
\hline Blanco & 49 & $3,053 \mathrm{a}$ & 11,210 & Blanco & 41 & $2,681 \mathrm{~b}$ & 7,987 \\
\hline Mezcla & 52 & $2,174 \mathrm{a}$ & 6,684 & Mezcla & 49 & $2,584 \mathrm{~b}$ & 26,533 \\
\hline
\end{tabular}

Letras iguales en la misma columna indican que las medias son iguales.

En cuanto al tiempo de consumo, se obtuvo para la presentación en fresco diferencia altamente significativa $(p<0.01)$ en el efecto color y especie; en la presentación seca los resultados muestran que hubo diferencia altamente significativa $(p<0.01)$ para el efecto especie, contrario al color que no hubo diferencia significativa $(p>0.05)$ (Tabla 3$)$. 
Tabla 3. ANOVA para tiempo de consumo en minutos acorde con color y especie para Hibiscus rosa - sinensis, Bougainvillea glabra, loxa cocinea, Rosa sp, Dianthus $s p .$, Aster spp variedades rojo, amarillo y blanco.

\begin{tabular}{|c|c|c|c|c|c|c|}
\hline \multicolumn{7}{|c|}{ Fresco } \\
\hline FV & GL & CM & $\mathbf{F}$ & $F_{0.05}$ & $F_{0.01}$ & \\
\hline Color & 3 & 2528,511 & 7,295 & 2,646 & 3,88 & ** \\
\hline Especie & 5 & 32681,741 & 94,295 & 2,255 & 3,11 & * \\
\hline \multicolumn{7}{|c|}{ Seco } \\
\hline FV & GL & CM & $F$ & $F_{0.05}$ & $F_{0.01}$ & \\
\hline Color & 3 & 668,560 & 0,933 & 2,646 & 3,88 & NS \\
\hline Especie & 5 & 25431,647 & 35,501 & 2,255 & 3,11 & $* *$ \\
\hline
\end{tabular}

Mediante prueba de Tukey, se pudo analizar que al tener tres colores (rojo, amarillo y blanco) el menor tiempo de consumo fue para el color rojo, seguido por la mezcla de estos colores, luego el amarillo y por último el blanco. Cuando se tenían solo dos colores, rojo y amarillo, la mezcla de estos dos colores registraba el menor tiempo, seguido por el rojo y luego por el amarillo. En ambos casos se evidencia que el amarillo es estadísticamente diferente en relación al rojo y a las mezclas (Tabla 4).

Tabla 4. Prueba de Tukey para tiempo de consumo por color en minutos.

\begin{tabular}{|l|l|l|l|l|c|}
\hline Color & $\mathbf{n}$ & \multicolumn{1}{|c|}{$\mathbf{X}$} & Color & $\mathbf{n}$ & $\overline{\mathbf{X}}$ \\
\hline Blanco & 60 & $52,194_{\mathrm{a}}$ & Amarillo & 40 & $38,169_{\mathrm{b}}$ \\
\hline Amarillo & 60 & $50,623_{\mathrm{a}}$ & Rojo & 40 & $69,309_{\mathrm{b}}$ \\
\hline Mezcla & 60 & $43,663_{\mathrm{ab}}$ & Mezcla & 40 & $66,927_{\mathrm{b}}$ \\
\hline Rojo & 60 & $38,169_{\mathrm{b}}$ & & & \\
\hline
\end{tabular}

Letras iguales en la misma columna indican que las medias son iguales

\section{Discusión}

Con la variable tiempo de acceso al alimento se evaluó el grado de atracción que ejercen las flores seleccionadas sobre G. carbonaria, al no haber diferencias significativas se puede determinar, que especie y color no son los únicos factores que pueden influir en esta variable, sino que pueden relacionarse otras características como lo señalan CASTAÑO y LUGO (1979), quienes afirman que $G$. carbonaria para hallar la comida se guían por el olfato y la vista.

Además, que el tiempo de acceso al alimento no presente diferencias significativas en relación con los colores y la especie puede relacionarse con la condición omnívora que le permite alimentarse de diferentes tipos de dietas en cualquier condición. G. carbonaria tiene la tendencia a ser oportunista en su alimentación, lo cual queda demostrado en los estudios que para medio natural señalan diversas preferencias de consumo, que van desde frutos maduros, hierbas, tubérculos, 
hongos, carroña e insectos, entre otros (CASTAÑO y LUGO, 1979; PRITCHARD y TREBBAU, 1984; MERCHAN et al., 1998).

La inexistencia de diferencias significativas en cuanto al tiempo de acceso al alimento, muestra la existencia de un comportamiento que pone de manifiesto una conducta social de ocurrencia en cautiverio, que puede asumirse como una consecuencia de la restricción espacial y el acostumbramiento al alimento o a las labores de manejo, lo que está acorde con los planteamientos de COLVÉE (2002), quien señala que en cautiverio $G$. carbonaria, posee una socialización a nivel de grupo que se manifiesta como un patrón que contradice el comportamiento general que se observa en medio natural, en donde es significativamente solitaria, juntándose solo bajo restricciones de alimento o agua o en época de apareamiento, aspecto con el cual también concuerdan los trabajos de MEDEM et al. (1979); CASTAÑO y LUGO (1979).

En cuanto a la presentación y el tiempo de consumo, se detecta significativamente que los alimentos frescos son preferidos, mientras que los alimentos secos, en donde hay pérdida y homogenización de la coloración se da preferencia por la especie ofrecida, lo que tendría que ver con la palatabilidad como lo señalan MERCADO y PALACIOS (2003).

Los diferentes tiempos de consumo obtenidos respecto al color del alimento ofrecido muestran que $G$. carbonaria diferencia los colores, siendo en orden de preferencia más apetecido el rojo, seguido del amarillo y el blanco; específicamente en este caso se da preferencia por las flores rojas, independientemente de la especie, lo anterior concuerda con lo reportado por CASTAÑO y LUGO (1979), PRITCHARD y TREBBAU (1984), quienes por propias observaciones y estudios realizados, afirman que $G$. carbonaria prefiere los alimentos de colores rojo y amarillo. Esto se contradice con los hallazgos de MERCADO y PALACIOS (2003), quienes en un trabajo anterior no encontraron diferencias significativas en la preferencia por el color de las flores ofrecidas; aunque si demostraron que había diferencia significativa en la palatabilidad.

La preferencia de los colores se puede explicar por la morfología interna del ojo, un perfeccionamiento muy importante en los quelonios, en donde se muestra que presentan conos más numerosos que se relacionan con agudeza visual y diferenciación de colores, además poseen unas pequeñas gotas de grasa de color cuya función puede ser filtrar la luz para recibir uno de los colores básicos, según lo plantean WEICHERT et al. (1985).

Señalan GIURFA y VOROBYEV (1998), GIURFA et al. (1996, 1997, 1999), que la orientación visual hacia el objeto es guiada no solamente por la visión en color sino también por la visión acromática; el sistema acromático es exclusivamente operado por los fotorreceptores de larga onda de longitud (señales de receptor L); mientras 
que la visión cromática posee diferentes resoluciones espaciales, entonces dependiendo de la distancia a la cual se halle el alimento y el ángulo en que se localice marcará proporcionalmente la influencia del color.

Como las señales acromáticas poseen gran resolución espacial y son detectadas desde largas distancias en comparación con la señales de color o cromáticas (GALIZIA et al., 2004), se puede asumir que la visión acromática pudo guiar el acercamiento al alimento, además de otras características como el olor y el acostumbramiento. Esta pudiera ser la razón de que no se detectaron diferencias significativas; pero en cuanto al tiempo de consumo en el cual se expresa significativamente la influencia del color, se puede aducir que la visón cromática señalada por WEICHERT et al. (1985), posiblemente fue la que marcó la diferencia. También hay que denotar que tanto el tamaño de la flor, aspecto que no fue medido en este ensayo, y si es cromática o acromática puede definir la distancia desde la cual puede ser observada según los planteamientos de GIURFA et al. (1996).

\section{Referencias}

CASTAÑO-MORA, O.V.; LUGO-RÚGELES, M. 1979. Estudio comparativo del comportamiento de dos especies de morrocoy, Geochelone denticulata y Geochelone carbonaria y algunos aspectos de su morfología externa. Trabajo de grado, Facultad de Ciencias. Departamento de Biología. Universidad Nacional de Colombia. Bogotá, D. E.

COLVÉE, S. 2002. Geochelone carbonaria, aspectos reproductivos de la tortuga de patas rojas en cautiverio. Reptilia 34:58-68.

DE LA OSSA, V.J. 1999. Zoocría: Guías académicos introductorias con énfasis en algunos reptiles. Universidad de sucre, Fac. de Ciencias Agropecuarias, Zootecnia. Sincelejo, Sucre, Colombia.

FREIBERG, M.A. 1971. El mundo de las tortugas. Editorial Albatros. Buenos Aires.

FRETEY, J. 1977. Les chéloniens de Guyane française. Étude préliminaire. Thesis, Universidade de Paris, France.

GALIZIA, G.C.; KUNZE, J.; GUMBERT, A.; BORG-KARLSON, A.K.; SACHSE, S.; MARKL, C.; MENZEL, R. 2004. Relationship of visual and olfactory signal parameters in a food-deceptive flower mimicry system. Behavioral Ecology 116 (1):159-168.

GIURFA, M.; VOROBYEV, M. 1998. The angular range of achromatic target detection by honey bees. Journal of Comparative Physiology 183:101-110.

GIURFA. M.; VOROBYEV, M.; KEVAN, P.; MENZEL, R. 1996. Detection of coloured stimuli by honeybees: minimum visual angles and receptor specific contrasts. Journal of Comparative Physiology 178:699-709. 
GIURFA, M.; VOROBYEV, M.; BRANDT, R.; POSNER, B.; MENZEL, R. 1997. Discrimination of coloured stimuli by honeybees: alternative use of achromatic and chromatic signals. Journal of Comparative Physiology 180:235-243.

GIURFA, M.; ZACCARDI, G.; VOROBYEV, M. 1999. How bees detect coloured targets using different regions of their compound eyes. Journal of Comparative Physiology 185:591-600.

HNATIUK, S.H. 1978. Plant dispersal by the Aldabran giant tortoise, Geochelone gigantea (Schweigger). Oecologia 36(3):345-350.

LEGLER, J.M. 1963. Tortoises (Geochelone carbonaria) in Panama: Distribution and Variation. American Midland Naturalist 70(2):490-503.

MEDEM, F. 1962. La distribución geográfica y ecológica de los Crocodylia y Testudinata en el Departamento del Chocó. Revista de la Academia Colombiana de Ciencias Exactas, Físicas y Naturales 11(44):279-342.

MEDEM. F.; CASTAÑO-MORA, O.; LUGO-RÚGELES, M. 1979. Contribución al conocimiento sobre la reproducción y el crecimiento de los morrocoyes (Geochelone carbonaria y Geochelone denticulata, Testudines, Testudinidae). Caldasia 12:497511.

MERCADO, E.; PALACIOS, R. 2003. Determinación de los patrones más relevantes de comportamiento reproductivo y alimenticio de Geochelone carbonaria bajo condiciones de manejo ex situ. Trabajo de Grado, Facultad de Ciencias Agropecuarias, Zootecnia. Universidad de Sucre.

MERCHAN, M.; FIDALGO, A.; PÉREZ, C. 1998. Biología, distribución y conservación del morrocoy o tortuga carbonera en los llanos de Venezuela. Reptilia 4(15):30-38.

MILTON, S.J. 1992. Plants eaten and dispersed by adult leopard tortoises Geochelone pardalis (Reptilia: Chelonii) in the southern Karoo. South African Journal of Zoology 27(2):45-49.

MOSKOVITS, D.K. 1981. Sexual dimorphism and population estimates of the Amazonian tortoises ( $G$. carbonaria and $G$. denticulate) in northwestern Brazil. Herpetologica 44(2):237-256.

PRITCHARD, P.C.; TREBBAU. U.P. 1984. Turtles of Venezuela. Soc. Study Amphib. Rept.. Venezuela.

STRONG, J.N.; FRAGOSO, M.V.J. 2006. Seed Dispersal by Geochelone carbonaria and Geochelone denticulata in Northwestern Brazil. Biotropica 38(5):683-686.

VARELA, R.O.; BUCHER, E.H. 2002 Seed Dispersal by Chelonoidis chilensis in the Chaco Dry Woodland of Argentina. Journal of Herpetology 36(1):137-140. 
WEICHERT, C.K.; PRESCH, W. 1985. Elementos de anatomía de los cordados. Editorial Mc Graw Hill. $3^{\mathrm{a}}$ ed. New York, USA.

ZAR, J.H. 1996. Bioestatistical analysis. Third edition. Prentice-Hall, Inc. Englewood Cliff, N.J. 